\title{
Une pratique de la pharmacie fondée sur des données probantes?
}

\author{
par Clarence Chant
}

$\mathrm{D}_{\mathrm{p}}^{\mathrm{s}}$ epuis leur apparition, les soins fondés sur des données probantes (SFDP) sont devenus la pierre angulaire de la formation en médecine, en pharmacie, en soins infirmiers et dans d'autres disciplines de la santé de même que de la pratique clinique quotidienne. Les programmes de pharmacie contiennent bon nombre de cours en matière de SFDP, ainsi que des cours en évaluation critique et en statistique. De plus, les principes des SFDP sont appliqués dans l'ensemble des cours en pharmacothérapie et des stages (au moyen de clubs de lecture et de présentations de cas). On pourrait croire instinctivement qu'il se passe la même chose dans la pratique de la pharmacie, à savoir que seules les activités s'appuyant sur des données probantes sont réalisées par les pharmaciens et les techniciens en pharmacie, particulièrement dans le contexte actuel où les ressources sont limitées. Malheureusement, on trouve facilement des exemples dans lesquels cette hypothèse pourrait être remise en question; c'est notamment le cas du bilan comparatif des médicaments. Des organismes de sécurité influents, comme l'Institut pour l'utilisation sécuritaire des médicaments au Canada (ISMP Canada) et Agrément Canada, ont beaucoup vanté les mérites du bilan comparatif des médicaments, l'annonçant comme une importante initiative au profit de la sécurité des patients ${ }^{1,2}$. En effet, d'ici 2020, Agrément Canada s'attend à ce que le bilan comparatif des médicaments soit réalisé et consigné pour tous les patients hospitalisés à chaque point de transition et qu'il soit aussi réalisé et consigné pour des sous-groupes choisis de patients ambulatoires ou de patients au service des urgences ne requérant pas une hospitalisation ${ }^{3}$.

Dans le présent numéro du Journal canadien de la pharmacie hospitalière (JCPH), l'étude par Duffet et collab. ${ }^{4}$ et la chronique «Le pour et le contre " ${ }^{5,6}$ portent sur la prophylaxie de l'ulcère de stress dans l'unité de soins intensifs (USI), un sujet qui a été bien approfondi grâce à des études rigoureuses et une pratique qui est largement admise comme norme dans la plupart des USI. Or, des chercheurs comme Deborah Cook, coauteure dans l'étude de Duffet qui a publié bon nombre d'articles phares sur le sujet, continuent d'évaluer les données publiées récemment afin de mieux comparer les risques et les avantages de ce traitement peu coûteux mais pas totalement bénin. Cependant, les données appuyant un bilan comparatif des médicaments exhaustif, une tâche à laquelle bon nombre de pharmaciens, de techniciens, de praticiens d'autres disciplines en santé et d'administrateurs ont consacré d'innombrables heures, ne font pas le poids face aux ressources qu'on investit dans le processus. Dans deux métaanalyses récentes, on a remarqué que les programmes de bilan comparatif des médicaments dirigés par des pharmaciens réduisaient les divergences relatives aux médicaments, mais pour ce qui est des divergences cliniquement significatives, les avantages étaient variables ou inexistants ${ }^{7,8}$. De plus, ces études ne montrent aucun effet sur des résultats plus concrets, comme les taux de mortalité, les taux de réadmission et les préjudices provoqués par les médicaments (ou par l'omission d'un traitement médicamenteux). En fait, pour bon nombre d'essais comparatifs à répartition aléatoire dans les méta-analyses de 2016,8, le groupe expérimental incluait un « ensemble » de fonctions du pharmacien, dont le bilan comparatif des médicaments. Par exemple, dans l'étude souvent citée de Gillespie et collab.', l'intervention comprenait les conseils sur les médicaments, une évaluation des médicaments pendant l'admission du patient et un suivi téléphonique après le congé. On peut avancer que s'assurer que le patient prend le bon médicament pour la bonne indication à l'aide d'une évaluation des médicaments est plus important et plus susceptible d'avoir mené à des résultats positifs que simplement évaluer lors des transferts de soins s'il y a lieu de continuer les médicaments pris à la maison par le patient. En d'autres termes, s'il n'y a pas d'indication valide, alors établir un bilan comparatif et poursuivre la prise d'un médicament sans évaluation critique pourrait être préjudiciable pour le patient! Enfin, dans la dernière version électronique de la boîte à outils du bilan comparatif des médicaments publié par ISMP Canada, on ne mentionne pas le bilan comparatif des médicaments et ses avantages concernant ces résultats « concrets $»^{2}$. 
Évidemment, le bilan comparatif des médicaments occupe un rôle important, mais la science n’a pas encore déterminé plusieurs des aspects clés, notamment : quand et comment réaliser le bilan comparatif des médicaments et pour qui et par qui doit-il être effectué afin d'atteindre le meilleur rapport coûtefficacité. Il s'agit des mêmes questions que nous posons pour toutes autres pharmacothérapies selon les principes des SFDP. Pour beaucoup de patients ayant besoin de prendre un grand nombre de médicaments (tels que les insuffisants rénaux chroniques ou ceux ayant subi une greffe d'organe), établir le meilleur schéma thérapeutique possible et vérifier la pharmacothérapie à l'aide d'une source secondaire, la meilleure pratique encouragée par ISMP Canada ${ }^{10}$, exige beaucoup de temps. Et il ne s'agit là que de la première étape du processus de bilan comparatif des médicaments! Réaliser efficacement cette tâche chronophage pour chaque patient et à chaque point de transfert ne correspond pas aux données probantes présentement disponibles. Par contre, il existe des données en faveur de sept autres activités cliniques associées à une réduction des taux de mortalité. Cela dit, ces autres activités ne sont pas effectuées par l'ensemble des pharmaciens pour tous les patients admissibles ${ }^{11}$. Cette dissonance semble difficile à justifier d'un point de vue scientifique s'appuyant sur des données probantes.

Mon intention n'est pas d'encourager l'abandon du bilan comparatif des médicaments, car je crois qu'il a un rôle à jouer lorsqu'il est employé à bon escient; considérez plutôt cet éditorial comme une exhortation à produire plus de données convaincantes permettant de mieux définir où et comment le bilan comparatif des médicaments s'inscrit dans l'arsenal du pharmacien. En effet, l'étude par MacDonald et collab. ${ }^{12}$ dans le présent numéro du $\mathrm{JCPH}$, qui s'intéresse au moyen le plus efficace d'obtenir un meilleur schéma thérapeutique possible pour les patients dans un service des urgences très fréquenté ne requérant pas une hospitalisation, sera un ajout bien nécessaire à la littérature sur le sujet. Lorsque nous aborderons notre pratique et l'adoption de normes avec la même rigueur que nous apportons aux SFDP, alors seulement pourrons-nous réellement affirmer que nous exerçons en nous appuyant sur une approche fondée sur des données probantes!

\section{[Traduction par l'éditeur]}

\section{References}

1. Agrément Canada; Institut canadien d'information sur la santé; Institut canadien pour la sécurité des patients; Institut pour la sécurité des médicaments aux patients du Canada. Bilan comparatif des médicaments au Canada : hausser la barre. Progrès à ce jour et chemin à parcourir. Ottawa (ON) : Agrément Canada; 2012.
2. Electronic Medication Reconciliation Group. Boîte à outils pour le remplacement $d u$ bilan comparatif des médicaments sur papier par sa version électronique. $2^{\mathrm{e}}$ édition. Toronto (ON) : ISMP Canada, Institut canadien pour la sécurité des patients; 2017.

3. Bilan comparatif des médicaments. Dans : Livret sur les Pratiques organisationnelles requises 2016. Ottawa (ON) : Agrément Canada; 2015. pp. 1 à16.

4. Duffet M, Choong K, Foster J, Gilfoyle E, Lacroix J, Cook DJ. Need for a randomized controlled trial of stress ulcer prophylaxis in critically ill children: a Canadian survey. Can J Hosp Pharm. 2017;70(4):288-93.

5. Yamashita S. Should we continue to use stress ulcer prophylaxis for critically ill patients? Le pour. Can J Hosp Pharm. 2017;70(4):316-7.

6. Duffet M. Should we continue to use stress ulcer prophylaxis for critically ill patients? Le contre. Can J Hosp Pharm. 2017;70(4):317-9.

7. Mekonnen AB, Abebe TB, McLachlan AJ, Brien JE. Impact of electronic medication reconciliation interventions on medication discrepancies at hospital transitions: a systematic review and meta-analysis. BMC Med Inform Decis Mak. 2016;16:112-26.

8. Mekonnen AB, McLachlan AJ, Brien JE. Effectiveness of pharmacist-led medication reconciliation programmes on clinical outcomes at hospital transitions: a systematic review and meta-analysis. BMJ Open. 2016;6: e010003.

9. Gillespie U, Alassaad A, Henrohn D, Garmo H, Hammarlund-Udenaes M, Toss $\mathrm{H}$, et al. A comprehensive pharmacist intervention to reduce morbidity in patients 80 years or older: a randomized controlled trial. Arch Intern Med. 2009;169(9):894-900.

10. Medication reconciliation (MedRec): about MedRec. Toronto (ON) : Institut pour la sécurité des médicaments aux patients du Canada. Publié au : https://www.ismp-canada.org/medrec/. Consulté le 23 juin 2017.

11. Bond CA, Raehl CL. Clinical pharmacy services, pharmacy staffing, and hospital mortality rates. Pharmacotherapy. 2007;27(4):481-93.

12. MacDonald N, Manuel L, Brennan H, Musgrave E, Wanbon R, Stoica G. Reliability of best possible medication histories completed by non-admitted patients in the emergency department. Can J Hosp Pharm. 2017;70(4):263-9.

Clarence Chant, Pharm. D., BCPS, FCCP, FCSHP, est directeur de pharmacie du St Michael's Hospital, à Toronto, en Ontario. Il est également rédacteur adjoint au Journal canadien de la pharmacie hospitalière.

Intérêts concurrents : Aucun déclaré.

Address correspondence to:

$D^{r}$ Clarence Chant

Service de pharmacie

St Michael's Hospital

30 Bond Street, Room B0007

Toronto ON M5B 1W8

Courriel : chantc@smh.ca 